\title{
Exploring the interaction of executive function and language processing in adult cognitive- communication disorders
}

Bijoyaa Mohapatra

Department of Communication Disorders, New Mexico State University, Las Cruces, New Mexico, USA

Purpose: The neural basis of language processing partially overlaps with executive system processing mediated by a network of frontal, parietal, and subcortical areas of the brain, thereby suggesting the significant interaction of executive functioning on linguistic abilities. It is critical that speech language pathologists understand the nature of executive function (EF) and their relationship with respect to speech and language. The purpose of this paper is to analyze the literature and evaluate the role of EF on language processing and deficits in communication disorders.

Methods: The following paper is a comprehensive literature review of the existing evidence regarding the influence of EF on language processing in adult neurogenic cognitive-communication disordered populations. The review includes relevant research studies from participants of all age groups and severities published in the last three decades.

Results: Research evidence with relevance to major adult neurogenic cognitive-communication disorders including Aphasia, Traumatic Brain Injury, Right Hemisphere Damage, and Parkinson's Disease are discussed. The paper addresses how the impact may vary with respect to the different components of language (semantics versus discourse pragmatics), different modalities of language (production versus comprehension), as well as complexities of language tasks. Several research studies provide support for the influence of different EF processes such as switching, shifting, inhibition, sequencing, working memory, and processing speed, on language processing in these clinical population.

Conclusions: Through the information presented here, professionals, both clinicians and researchers in the field of communication sciences and disorders can gain a thorough knowledge of the interaction of language and executive functioning and a prospect to develop an integrative framework for assessment and intervention of cognitive-communication disorders. Keywords: Executive functions, language, semantics, pragmatics, neurogenic communication disorders

\section{INTRODUCTION}

Communication enables speakers and listeners to organize their thoughts into spoken output based on their internal goals and a desire to express. An individual depends heavily on the sensory and memory systems to accomplish tasks, such as choosing the right word that fits in the context of a conversation, judging a sentence for its grammaticality, and switching from one language to another. With the rich source of information

(C) 2019 The Korean Association of SpeechLanguage Pathologists

This is an Open Access article distributed under the terms of the Creative Commons Attribution NonCommercial license (http://creativecommons.org/ licenses/by-nc/4.0/) which permits unrestricted noncommercial use, distribution, and reproduction in any medium, provided the original work is properly cited. 
and plenty of behavioral alternatives, there is a high probability of conflicts and interferences that occur during linguistic processing. In order to manage these conflicts and interferences effectively, human beings recruit executive processes to monitor and regulate linguistic expressions to match their internal goals [1,2]. Therefore, the ability to communicate relies not only upon an intact language system, but also on higher order control mechanisms such as executive function (EF) that are recruited in language processing. Executive function (a collective term for all executive processes) involves highlevel goal-directed behavior that encompasses many cognitive control operations, such as, strategic planning of goal-directed action, inhibition of irrelevant responses, monitoring and regulating of behavior, processing, organizational skills, decision-making, and problem solving [3-5].

Baddeley and Hitch proposed a conceptual model of working memory (WM) [6] that consists of a limited capacity attentional control system (central executive) aided by two slave systems, the phonological loop, and the visuospatial sketchpad. The central executive encompasses a complex set of control mechanisms that underlie goal-oriented decisions and cognitive flexibility. It is fractionated into different executive subprocesses $[7,8]$ that are typically involved in functions (referred to as the EFs), such as, inhibition of irrelevant information, maintaining and shifting between tasks or sets, attending selectively to different stimuli, goal-setting, planning, and organizing. The WM model has a significant role in language processing, in the form of the phonological loop that supports rehearsal and storage of verbal and acoustic information and interacts with the long-term memory via the multimodal episodic buffer (a fourth component of the WM model added later in an updated model by Baddeley). Several researchers have viewed $\mathrm{EF}$ and $\mathrm{WM}$ as multidimensional cognitive constructs and have documented strong relationship with different aspects of language, learning, and comprehension $[9,10]$.

\section{ROLE OF EF IN LANGUAGE PROCESSING}

Executive function plays a crucial role in setting and accomplishing verbal acts, such as, oral and written production, deactivation of task-irrelevant lexical representations, monitoring of ongoing behavior, and selection among competing sentential representations. Speakers utilize EF to choose the right word over other competing options and refrain from generating an improper word [11]. They also utilize executive processes to regulate the ability to switch between two languages or bilingual language use [12]. Additionally, listeners require EF to select and grasp relevant information from a speech output by focusing on the meaning and ignoring what they already know i.e. preconceived notions [13].

During sentence comprehension, EFs are also used to monitor and select judiciously among competing sentence representations. Conflict inhibition plays a major role during this and allows for a coherent interpretation of the sentence. Adults with poor comprehension skills are likely to demonstrate deficits in the inhibitory mechanism and suppression of competing representations. With respect to syntactically ambiguous sentences, the speaker's ability to control conflicts is often confounded by the linguistic elements and format of complex sentence structures [14]. However, executive processes such as error detection and cognitive monitoring trigger control mechanisms to resolve conflicts and aid in better comprehension. Similarly, conflict control in word production is contributed by executive processes such as, inhibition of prepotent responses, controlled access to lexical items in semantic memory, and attention control to resolve competitions among lexical representations $[11,15]$. Hence, it is critical for speech language pathologists to understand the nature of EF and their relationship with respect to speech and language. The purpose of this paper is to provide an overview of the existing literature on the role of $\mathrm{EF}$ in language processing (and deficits) in communication disorders.

\section{LANGUAGE AND EF DEFICITS IN COGNITIVE- COMMUNICATION DISORDERS}

The following section provides an account of the research examining the link between EF and language abilities in adult neurogenic cognitive-communication disorders.

Specific terms that included, but not restricted to, executive function/s, switching, shifting, inhibition, sequencing, working memory, processing speed, language, verbal, non-verbal, communication, comprehension, expression, fluency, naming, functional communication, aphasia, traumatic brain injury, right hemisphere damage, and Parkinson's disease were searched on the Google Scholar database. All age groups and severity ranges were considered, and the search period spanned for over three decades beginning from 1980. All studies were scanned for relevancy with respect to discussion on the interaction of language and EF abilities and subsequently included in the review. Table 1 provides a summary of the language and EF dimensions that have already been researched in the 
Table 1. Summary of language and executive function dimensions already researched in the clinical population

\begin{tabular}{|c|c|c|c|}
\hline Clinical population & Language dimensions studied & Executive function dimensions studied & Published literature* \\
\hline \multirow[t]{7}{*}{ Aphasia } & Naming & Inhibition & $16,17,18,19,20,22$ \\
\hline & Semantics & Shifting & $27,28,29,30,31,32$ \\
\hline & Auditory processing & Divided attention & $34,35,36$ \\
\hline & Verbal production & Cognitive control & \\
\hline & Conversation & & \\
\hline & Picture-description & & \\
\hline & Language comprehension & & \\
\hline \multirow[t]{6}{*}{ Traumatic Brain Injury } & Pragmatics & Planning & $42,43,44,45,46,47$ \\
\hline & Conversation & Self-monitoring & $48,49,50,51,52,53$ \\
\hline & Narrative discourse & Switching & $54,55,56,57,58,59$ \\
\hline & Story retelling & Inhibition & \\
\hline & Story grammar production & Cognitive flexibility & \\
\hline & Procedural description & & \\
\hline \multirow[t]{7}{*}{ Right Hemisphere Damage } & Narration & Shifting & $34,59,68,69,70,71$ \\
\hline & Pragmatics & Processing speed & \\
\hline & Language comprehension & Planning & \\
\hline & Language production & & \\
\hline & Semantic and phonemic verbal fluency & & \\
\hline & Metaphor interpretation & & \\
\hline & Discourse production & & \\
\hline \multirow[t]{5}{*}{ Parkinson's disease } & Sentence comprehension & Cognitive sequencing & $76,77,78,79,80,81$ \\
\hline & Emotional prosody & Set-switching & 82,83 \\
\hline & Conversation & Working memory & \\
\hline & & Response inhibition & \\
\hline & & Selective attention & \\
\hline
\end{tabular}

*Numbers in this column correspond with the references in the manuscript.

clinical populations. A narrative review of the specific findings in each clinical population is presented in the following paragraphs.

\section{Aphasia}

Several studies in aphasia have investigated the nature of cognitive processing deficits during speech production, and reported, prolonged inhibition on naming tasks [16], inability to control active representations within the lexical system [17], interferences in selection of word due to damage in syntactic control processes [18], and poor inhibitory function in semantic short-term-memory (STM) deficits [19]. Often these studies have employed semantically-blocked picture naming tasks where repeated blocks of items from the same semantic category are presented to the participant, and the response on these items are compared with the response obtained from mixed blocks (where repeated items from semantically unrelated categories are presented). Studies have shown that individuals with aphasia (IWA) demonstrate increased naming latencies on the semantically blocked items $[16,17]$ and this effect is exaggerated when the repetitions are presented at a faster rate. McCarthy and Kartsounis attributed this semantic blocking effect to excessive inhibition of lexical representations which can be explained as follows: after one complete phase of speech production, lexical representations are inhibited to prevent their reselection into spoken vocabulary again. For semantically related items, there is a chance of exaggerated inhibition due to the competition between similar representations. This effect is likely to be magnified in individuals with brain damage [20]. Wilshire and McCarthy argued against such explanation and suggested that increased naming latencies on semantically blocked items may be due to 
stronger co-activation of two lexical representations, to the extent that one representation competes over the nearest representation, which leads to prolonged latencies in verbal production. In another study, the effect of inhibition deficits on word production and comprehension was investigated in fluent and nonfluent IWA on picture-naming and word-picture matching tasks. Non-fluent participants showed semantic blocking effects in both tasks but the effects were more prominent on the naming task [20]. This finding further strengthened the result that the semantic blocking effect is prominent in lexical (naming) tasks compared to semantic selection (matching) tasks. The authors suggest that speech production in non-fluent aphasia is a result of the prolonged activation of lexical representations primarily due to post selection inhibition. Research has also shown that the inability to retain semantic information in the case of persons with limited STM may be due to inhibitory deficits in $\mathrm{EF}$ [21]. In another experiment conducted by Martin and colleagues, patients with semantic STM deficits showed difficulty in inhibiting distractor words. They showed greater interference in reaction times and latencies on the items that were related semantically when compared with items that were unrelated. Such difference in reaction times could be due to the greater competition in the semantically related words (i.e. choosing between items that are semantically related is more difficult than unrelated items), and to impaired inhibition of competing words within the semantically related word lists. Authors explained this by associating it with the inability to inhibit lexical-semantic representations.

Another relatively less explored area in aphasia is the relationship between EF and conversation. Conversation involves complex cognitive processes such as, planning, organization, monitoring and sequencing; therefore, deficits to any of these executive processes may sometimes result in conversational deficits. In a case study of an adult female with aphasia, Frankel and colleagues compared her conversational deficits to EF deficits recorded on a test battery [22]. The inability to shift between topics and take turns during conversation was associated with deficits in shifting and divided attention, and the presence of perseverations in conversation was indication of her deficits in response inhibition. Deficits in (verbal) WM also resulted in impaired topic management, topic maintenance, and topic shifting in her conversation. Similar deficits in EFs (shifting and inhibition) were confirmed from the Trail Making Test (TMT) [23] and Wisconsin Card Sorting Test (WCST; [24], and visual and strategic memory on the Self Or- dering Pointing Test [25]. Difficulty in forming concepts was reflected in the patient's high frequency of perseverations during speech. This demonstrated her inability to shift from limited forms of expression to cognitively flexible and relevant forms of communication. She also exhibited difficulty in strategy-generation by failing to produce novel material to meet the conversational demands. This executive (strategy-generation) deficit was evidenced on the Five-Point Test and Design Fluency task [26]. Overall, this study is a classic example that accounts for the different EFs involved in communication, specifically during conversation.

Other investigators have provided evidence for the detrimental effects of divided attention on language abilities like auditory processing [27], phoneme and semantic judgment [28], grammaticality judgments [29], and semantic classification and lexical decision [30]. Murray and colleagues investigated the speech production ability in individuals with mild aphasia [31]. Participants performed on dual tasks: picturedescription and tone-discrimination tasks and results suggested that IWA produced shorter and less complex utterances with change in task conditions (from single task to divided attention), thereby making the overall communication less effective.

Kuzmina and Weekes explored the interaction of cognitive control and language abilities in fluent and non-fluent aphasias using verbal and non-verbal cognitive control tasks. They found that all IWA were challenged by cognitive control tasks; but non-fluent IWA were vulnerable to both verbal and nonverbal tasks compared to the fluent IWA. Also, participants recruited different cognitive mechanisms for language comprehension versus picture naming tasks. They utilized non-verbal cognitive control for the former and verbal cognitive control during the later task [32]. Murray also examined the interaction of EF and language performance in participants with aphasia and right hemisphere brain damage using a design fluency task, the Ruff Figural Fluency Test (RFFT) [33], and found that EF deficits were significantly poorer for both the clinical groups in comparison to the controls and the EF performances correlated with other cognitive and language tests $[34,35]$. Similarly, Mohapatra and Marshall [36] investigated the different EFs from Baddeley's WM model including setswitching, updating, inhibition, and dual task processing, and found that IWA demonstrated more difficulties on higherlevel complex EF sections of the Color Trails Test [37] and nback task [38]. Aphasia severity on the Western Aphasia Battery-Revised [39] correlated with diminished performance on 
EF measures. Overall, the above studies provide evidence for a significant relationship between executive deficits and language abilities in IWA.

\section{Traumatic Brain Injury (TBI)}

Traumatic Brain Injury often affects areas in the fronto-basal and fronto-temporal regions of the brain [40] that are largely involved with EFs [41]. McDonald studied pragmatic difficulties in TBI and found that frontal lobe deficits exhibited by TBI also affects discourse abilities [42]. Problems in planning and self-monitoring behaviors may result in conversational discourse deficits [43] while disinhibition will reduce the ability to utilize clues/hints during conversation [44]. Furthermore, inefficiency to address pragmatic issues reflects deficiency in abstraction skills [45]. Other researchers report significant associations between verbal dysfluency and difficulties in switching between components [46], and pragmatic deficits and the inability to inhibit [47] in TBI. Ylvisaker and Szekeres attribute the communication deficits in children and adults with TBI to their impairments in EF on several dimensions including planning, self-awareness, self-inhibition, problem solving, and initiation [48]. Some studies have utilized narrative discourse analyses to investigate verbal communication deficits in TBI [49-52]. The difficulties in discourse production in TBI are described as setbacks in EF, particularly, to a decreased capacity to devise and accomplish a goal. A study conducted by Coelho and colleagues correlated the performance of EF with story retelling and story generation tasks [53]. Story structure utilizes several executive processes, such as, organization and production of a story and is made up of an episode that comprises of a goal, an endeavor to accomplish the goal, and an outcome that marks the accomplishment of the goal. In the above context, EF includes the capabilities required for defining a goal, devising a plan for its accomplishment, and successful execution of the plan. Results from the study suggested that the performance of the TBI participants on discourse analysis (in the form of story retelling and story generation) was highly correlated to their executive performance measured on the WCST.

Story grammar production is another method of assessing discourse performance. The key components of this method are narrative organization and developing coherence in the description of events and characters. Mozeiko et al. reported significant correlation between measures of story grammar and executive processes assessed on a sorting task [54]. They associated this relationship to the ability to form episodes which is a key component of storytelling and requires a great deal of cognitive flexibility. Other studies have investigated the relationship between EFs and pragmatic impairment in TBI [47,53,55-59]. These studies have utilized neuropsychological tests such as the Wechsler Memory Scale [60], the WCST, the Test of Everyday Attention [61], and the Rey Auditory Verbal Learning Task (RAVLT) [62] to assess inhibition, cognitive shifting, and concept formation. The pragmatic tasks employed in most of these studies constitute: story retelling, story generation, conversation, contextual meaning task, and procedural description. Zimmerman and colleagues assessed pragmatic deficits of TBI adults on a variety of tasks (conversational discourse, metaphor interpretation, narrative discourse, and indirect speech acts) and found positive correlation with a wide range of executive processes such as initiation, inhibition, planning, switching, and WM. Semantic verbal fluency task, a measure of the Montreal Communication Evaluation Battery [63] was the most affected in TBI participants; this finding was associated with difficulties in switching, one of the important EFs [46]. However, another study using the La Trobe Communication Questionnaire (LCQ) [64] suggested that a considerable fraction of pragmatic problems in TBI participants was represented by EF measures. The questionnaire (LCQ) used in this study allowed for assessment of conversational problems confronted by individuals with TBI and with their communication partners. The EF measures used were the F-A-S verbal fluency task (task management, initiation and cognitive flexibility; [65], the Speed and Capacity of Language Processing test (index of speed of verbal information processing; [66], and the RAVLT (measures the ability to manipulate information). Word retrieval and fluency (verbal) abilities obviously require EFs, including planning and monitoring, memory regulation and management, and attention allocation. Additionally, performance on the verbal fluency tasks emphasizes the ability to inhibit the usual retrieval strategy based on word meanings and adopt a retrieval strategy that depends greatly on sound-form representations of speech [67]. In summary, it is evident from the TBI literature that linguistic deficits (including narration) are consistent with impairments to EFs.

\section{Right Hemisphere Damage (RHD)}

Several research studies reveal the possible association of language impairment and EFs in RHD. Persons with RHD often face difficulties in developing themes and abstract concepts to organize a narrative, incorporating new information to the ex- 
isting [68], and using themes to organize stories [69]. They also demonstrate difficulties in interpretation of linguistic content such as humor and jokes [70] and lack in inference-making [71]. Such deficits are demonstrated to be consistent with damage to executive systems [45]. However, relatively limited literature is available on the relationship between pragmatic difficulties and EF in RHD. In a study by McDonald, Controlled Oral Word Association Test [72] and WAIS-R (Similarities subtest) [73] were used to demonstrate the relationship between pragmatic and executive abilities. Pragmatic abilities were assessed on comprehension (sarcasm and requests) and production (narrative, requests and conversation) tasks. In addition, a syllogism task was administered; sentences that require logical reasoning to deduce and solve are called syllogisms (an example from their study is "Electricity is a form of power; microwaves use electricity to run; do microwaves use power to run?"). While pragmatic abilities were not correlated with executive dysfunction, performance on syllogism tasks suggested some executive dysfunction in the clinical population (18 RHD participants were evaluated in the above study). Syllogisms require participants to disregard the literal meaning of the sentence but pay attention to the underlying meaning of the sentence. Thus, it challenged participants to ignore the semantic content and focus on the pragmatic content of the sentence. This ability to shift focus from one function to another is an executive ability and is referred to as switching.

In another study, Zimmerman and colleagues reported association between pragmatic and executive deficits in RHD participants. Various EF tests included the TMT, Hayling Test [74], WCST, semantic and phonemic verbal fluency tasks from Montreal Communication Evaluation Battery [63], and WM tasks from the Brazilian Brief Neuropsychological Assessment Battery Neupisilin [75]. Information on pragmatic processing was obtained from the Montreal Communication Evaluation Battery on tasks such as conversational discourse, metaphor interpretation, narrative discourse and indirect speech acts. Positive correlations were observed between EFs and pragmatic abilities. Processing of metaphors was associated with executive processes such as switching, WM and processing speed. Narrative discourse was strongly related to processing speed, planning, flexibility, and WM. Inaccurate discourse production was attributed to deficits in topic organization and discursive impairment in RHD participants [59] which is also an indication of the inability to suppress information. Besides this, executive dysfunction was also observed in the verbal WM and initiation processes. In summary, pragmatic deficits in persons with RHD were attributed to inhibitory and WM deficits.

\section{Parkinson's disease}

Often researchers suggest that executive dysfunction is the underlying reason for deficits in sentence comprehension in Parkinson's disease (PD). Other researchers attribute comprehension deficits to processes such as, set-switching $[76,77]$, sequencing [78], inhibition [79], verbal WM [78,80] and information processing speed $[81,82]$. A study by Colman et al. examined the relation between sentence comprehension and EFs in 28 Dutch speaking PD participants [83]. The sentence comprehension task involved comparing different phrasestructures (active versus passive) and varying sentence lengths (short versus long). Executive function was evaluated using TMT (parts A and B), Test Battery of Attentional Performances (TAP) [84], Odd Man Out Test [85), Stroop task [86], digit span subtest of the Wechsler Adult Intelligence scale (WAIS) [87] and Lelekov's protocol for cognitive sequencing [88]. Results from EF tasks suggested that persons with PD scored lower in set-switching (TMT, Odd Man Out Test) and sustained visual attention tasks (TAP) and showed no significant differences on other functions such as inhibition and WM. Sentence comprehension scores were similar with scores obtained from sustained visual attention tasks. In particular, deficits in set-switching were associated with deficits in comprehending passive sentences. This emerges from the fact that sentence comprehension requires a listener to switch between contending linguistic information, therefore, impairment in setswitching abilities is likely to contribute to difficulties in comprehension of passive sentences in PD participants.

The other EFs that have an effect on sentence comprehension are inhibition, $\mathrm{WM}$, and sequencing. Inhibition is strongly associated with set-switching and requires suppression of irrelevant linguistic information to process the required information. Working memory is responsible for storing and manipulating words and phrases in order to interpret phrasestructure or linguistic elements in a sentence (such as subjectobject-verb combinations and active/passive sentences). Moreover, sentence comprehension requires organizing the sequence (time and order) of information to build up coherence during communication, which is is an executive process. Overall, different EF processes demonstrate association with language comprehension and expression.

Emotional dysprosody (flat and nonemotional speech) is another characteristic feature of PD speech. Working memory 
and EF deficits are not independent of prosodic deficits observed in PD. Often prosodic deficits in the PD is visualized as a cognitive deficit rather than a linguistic deficit, because persons with PD frequently exhibit dysfunctions in the central executive component of Baddeley's WM model [89]. In a study by Breitenstein and colleagues, relationship between emotional prosody and central EF was explained. Twenty participants were assessed on a neuropsychological test battery consisting of WCST, Digit Span subtests of the Wechsler Adult Intelligence Scale-Revised (WAIS-R; [73] and Listening Span Test [90]. Two prosodic perception tasks were administered: one in English and the other in German language; the pitch and duration of the cues were altered for sentences. Results suggested that central executive functioning was significantly correlated with PD participants' performance on prosodic perception tasks. Processing emotional prosody during conversation demands a significant amount of attentional resources. For example, responding to verbal content requires a person to pay attention to the prosodic meaning of the sentence and inhibit the irrelevant semantic meaning that ultimately requires selective attention (or response inhibition). Both these processes are linked to the central executive of the WM system [91,92]. Thus, individuals with PD have difficulty in linguistic deficits that are often a consequence of executive dysfunction.

\section{SUMMARY}

This review summarizes findings from research studies exploring the relationship between executive functioning and linguistic abilities in different neurogenic communication disorders. Executive processes such as switching, shifting, inhibition, sequencing, verbal WM, and processing speed have a strong correlation with linguistic aspects of narration, story retelling, conversational discourse, metaphor interpretation, phonological processing and indirect speech acts. Based on the current review, it would be logical to conclude that there is significant contribution of EFs on linguistic abilities. It also provides support for overlap in the networks of language processing and executive system processing, mediated by a network of frontal, parietal, and subcortical areas of the brain [13]. In summary, the influence of executive control on language comprehension and production are multiple and must be accounted for in all speech and language related assessments and interventions. Future empirical efforts should incorporate $\mathrm{EF}$ training into rehabilitation programs for cognitive-communication disorders.

\section{REFERENCES}

1. Miller EK. The prefontral cortex and cognitive control. Nature reviews neuroscience. 2000;1:59-65.

2. Miller EK, Cohen JD. An integrative theory of prefrontal cortex function. Annual review of neuroscience. 2001;24:167-202.

3. Stuss DT, Knight RT. Principles of frontal lobe function: Oxford University Press; 2013.

4. Hull R, Martin RC, Beier ME, Lane D, Hamilton AC. Executive function in older adults: a structural equation modeling approach. Neuropsychology. 2008;22:508.

5. Chan RC, Shum D, Toulopoulou T, Chen EY. Assessment of executive functions: Review of instruments and identification of critical issues. Archives of Clinical Neuropsychology. 2008;23(2):201-16.

6. Baddeley AD, Hitch G. Working memory. Psychology of learning and motivation. 1974;8:47-89.

7. Baddeley A. Fractionating the central executive. Principles of frontal lobe function. 2002:246-260.

8. Shallice T. Fractionation of the supervisory system. Principles of frontal lobe function. 2002:261-277.

9. Murray LL. Attention and other cognitive deficits in aphasia: Presence and relation to language and communication measures. American Journal of Speech-Language Pathology. 2012;21:S51S64.

10. Esslinger PJ. Conceptualizing, describing, and measuring components of executive function: A summary. In: Lyon GR, Krasnegor NA, editors. Attention, memory, and executive function. Baltimore, MD: Paul Brookes; 1996. p. 367-395.

11. Badre $\mathrm{D}$, Wagner $\mathrm{AD}$. Left ventrolateral prefrontal cortex and the cognitive control of memory. Neuropsychologia. 2007;45:28832901.

12. Abutalebi J, Green D. Bilingual language production: The neurocognition of language representation and control. Journal of neurolinguistics. 2007;20:242-275.

13. Ye Z, Zhou X. Executive control in language processing. Neuroscience \& Biobehavioral Reviews. 2009;33:1168-1177.

14. Novick JM, Trueswell JC, Thompson-Schill SL. Cognitive control and parsing: Reexamining the role of Broca's area in sentence comprehension. Cognitive, Affective, \& Behavioral Neuroscience. 2005;5:263-281.

15. Thompson-Schill SL, Bedny M, Goldberg RF. The frontal lobes and the regulation of mental activity. Current opinion in neurobiology. 2005;15:219-224.

16. McCarthy RA, Kartsounis LD. Wobbly words: Refractory anomia with preserved semantics. Neurocase. 2000;6:487-497.

17. Wilshire CE, McCarthy RA. Evidence for a context-sensitive word retrieval disorder in a case of nonfluent aphasia. Cognitive Neuropsychology. 2002;19:165-186.

18. Schwartz MF, Hodgson C. A new multiword naming deficit: Evidence and interpretation. Cognitive Neuropsychology. 2002;19: 263-288.

19. Freedman ML, Martin RC, Biegler K. Semantic relatedness effects in conjoined noun phrase production: Implications for the role of 
short-term memory. Cognitive Neuropsychology. 2004;21:245-265.

20. Biegler KA, Crowther JE, Martin RC. Consequences of an inhibition deficit for word production and comprehension: Evidence from the semantic blocking paradigm. Cognitive neuropsychology. 2008;25:493-527.

21. Martin RC, Allen CM, editors. A disorder of executive function and its role in language processing. Seminars in speech and language; 2008: () Thieme Medical Publishers.

22. Frankel T, Penn C, Ormond-Brown D. Executive dysfunction as an explanatory basis for conversation symptoms of aphasia: A pilot study. Aphasiology. 2007;21:814-828.

23. Reitan RM. Trail Making Test: Manual for administration and scoring: Reitan Neuropsychology Laboratory; 1992.

24. Grant DA, Berg EA. Wisconsin Card Sorting Test (WCST): PAR.; 1993.

25. Petrides M, Milner B. Deficits on subject-ordered tasks after frontal-and temporal-lobe lesions in man. Neuropsychologia. 1982;20: 249-262.

26. Spreen O, Strauss E. A compendium of neurological tests. Administration, norms and commentary. 1998.

27. Murray LL, Holland AL, Beeson PM. Auditory Processing in Individuals With Mild AphasiaA Study of Resource Allocation. Journal of Speech, Language, and Hearing Research. 1997;40:792-808.

28. Tseng C-H, McNeil M, Milenkovic P. An investigation of attention allocation deficits in aphasia. Brain and language. 1993;45:276-296.

29. Murray LL, Holland AL, Beeson PM. Grammaticality judgements of mildly aphasic individuals under dual-task conditions. Aphasiology. 1997;11:993-1016.

30. Arvedson JC, McNeil MR. Accuracy and Response Times for Semantic Judgements and Lexical Decisions with Left-and RightHemisphere Lesions. Clinical Aphasiology 1986;17:188-200.

31. Murray LL, Holland AL, Beeson PM. Spoken language of individuals with mild fluent aphasia under focused and divided-attention conditions. Journal of Speech, Language, and Hearing Research. 1998;41:213-227.

32. Kuzmina E, Weekes BS. Role of cognitive control in language deficits in different types of aphasia. Aphasiology. 2017;31:765-792.

33. Ruff RM. RFFT: Ruff figural fluency test: Professional manual: Psychological Assessment Resources; 1996.

34. Murray LL. Design fluency subsequent to onset of aphasia: A distinct pattern of executive function difficulties? Aphasiology. 2017; 31:793-818.

35. Murray L. Focusing attention on executive functioning in Aphasia. Aphasiology. 2017;31: 721-724.

36. Mohapatra B, Marshall RS. Performance differences between aphasia and healthy aging on an executive function test battery. International Journal of Speech-Language Pathology. 2019:1-11.

37. D'Elia L, Satz P, Uchiyama C, White T. Color Trails Test. Odessa FL: Psychological Assessment Resources. Inc; 1996.

38. Wright HH, Downey RA, Gravier M, Love T, Shapiro LP. Processing distinct linguistic information types in working memory in aphasia. Aphasiology. 2007;21:802-813.

39. Kertesz A, editor. Western Aphasia Battery-Revised. SanAntonio,
TX: PsychCorp; 2007.

40. Mennel H. The morphology of traumatic head injury. Severe Head Injuries: Springer; 1997. p. 19-28.

41. Duncan J, Emslie H, Williams P, Johnson R, Freer C. Intelligence and the frontal lobe: The organization of goal-directed behavior. Cognitive psychology. 1996;30:257-303.

42. McDonald S. Pragmatic language skills after closed head injury: Ability to meet the informational needs of the listener. Brain and Language. 1993;44:28-46.

43. McDonald S, Pearce S. Requests that overcome listener reluctance: Impairment associated with executive dysfunction in brain injury. Brain and Language. 1998;61:88-104.

44. McDonald S, Pearce S. Clinical insights into pragmatic theory: Frontal lobe deficits and sarcasm. Brain and language. 1996;53:81104.

45. Martin I, McDonald S. Weak coherence, no theory of mind, or executive dysfunction? Solving the puzzle of pragmatic language disorders. Brain and language. 2003;85:451-466.

46. Kave G, Heled E, Vakil E, Agranov E. Which verbal fluency measure is most useful in demonstrating executive deficits after traumatic brain injury? Journal of clinical and experimental neuropsychology. 2011;33:358-365.

47. Channon S, Watts M. Pragmatic language interpretation after closed head injury: Relationship to executive functioning. Cognitive Neuropsychiatry. 2003;8:243-60.

48. Ylvisaker M, Szekeres SF. Metacognitive and executive impairments in head-injured children and adults. Topics in Language Disorders. 1989;9:34-49.

49. Tucker FM, Hanlon RE. Effects of mild traumatic brain injury on narrative discourse production. Brain injury. 1998;12:783-792.

50. Coelho C, Grela B, Corso M, Gamble A, Feinn R. Microlinguistic deficits in the narrative discourse of adults with traumatic brain injury. Brain Injury. 2005;19:1139-1145.

51. Body R, Parker M. Topic repetitiveness after traumatic brain injury: An emergent, jointly managed behaviour. Clinical linguistics \& phonetics. 2005;19:379-392.

52. Cannizzaro MS, Coelho CA. Analysis of narrative discourse structure as an ecologically relevant measure of executive function in adults. Journal of psycholinguistic research. 2013;42:527-549.

53. Coelho CA, Liles BZ, Duffy RJ. Impairments of discourse abilities and executive functions in traumatically brain-injured adults. Brain Injury. 1995;9:471-477.

54. Mozeiko J, Le K, Coelho C, Krueger F, Grafman J. The relationship of story grammar and executive function following TBI. Aphasiology. 2011;25:826-835.

55. Hartley LL, Jensen PJ. Narrative and procedural discourse after closed head injury. Brain Injury. 1991;5:267-285.

56. Coelho CA. Story Narratives of Adults With Closed Head Injury and Non-Brain-Injured AdultsInfluence of Socioeconomic Status, Elicitation Task, and Executive Functioning. Journal of Speech, Language, and Hearing Research. 2002;45:1232-1248.

57. Snow P, Douglas J, Ponsford J. Conversational discourse abilities following severe traumatic brain injury: A follow up study. Brain 
Injury. 1998;12:911-935.

58. Youse $\mathrm{K}$, Coelho C. Working memory and discourse production abilities following closed-head injury. Brain Injury. 2005;19:10011009.

59. Zimmermann N, Gindri G, Oliveira C, Fonseca RP. Pragmatic and executive functions in traumatic brain injury and right brain damage. Dementia \& Neuropsychologia. 2011;5:337-345.

60. Wechsler D. Wechsler Memory Scale (WMS-III): Psychological corporation; 1997.

61. Robertson IH, Ward T, Ridgeway V, Nimmo-Smith I. The test of everyday attention: TEA: Thames Valley Test Company Bury St. Edmunds,, UK; 1994.

62. Schmidt M. Rey auditory verbal learning test: A handbook: Western Psychological Services Los Angeles; 1996.

63. Fonseca RP, Parente M, Côté H, Ska B, Joanette Y. Bateria Montreal de avaliação da comunicação-bateria MAC. São Paulo: Pró-Fono. 2008.

64. Douglas JM, O'Flaherty CA, Snow PC. Measuring perception of communicative ability: The development and evaluation of the La Trobe Communication Questionnaire. Aphasiology. 2000;14:251268.

65. Spreen O, Benton AL. Neurosensory Center Comprehensive Examination for Aphasia: Manual of directions. Victoria, BC: Neuropsychology Laboratory, University of Victoria; 1969.

66. Baddeley AD, Emslie H, Nimmo-Smith I. The speed and capacity of language-processing test: Thames Valley Test Company; 1992.

67. Szatkowska I, Grabowska A, Szymañska O. Phonological and semantic fluencies are mediated by different regions of the prefrontal cortex. Acta neurobiologiae experimentalis. 2000;60:503-508.

68. Molloy R, Brownell HH, Gardner H. Discourse comprehension by right-hemisphere stroke patients: Deficits of prediction and revision. Discourse ability and brain damage: Springer; 1990. p. 113130.

69. Schneiderman EI, Murasugi KG, Saddy JD. Story arrangement ability in right brain-damaged patients. Brain and Language. 1992;43:107-120.

70. Bihrle AM, Brownell HH, Powelson JA, Gardner H. Comprehension of humorous and nonhumorous materials by left and right brain-damaged patients. Brain and Cognition. 1986;5:399-411.

71. McDonald S, Wales R. An investigation of the ability to process inferences in language following right hemisphere brain damage. Brain and Language. 1986;29:68-80.

72. Benton L, Hamsher K, Sivan A. Controlled oral word association test. Multilingual aphasia examination. 1994;3.

73. Wechsler D. Wechsler adult intelligence scale-revised: Psychological Corporation; 1981.

74. Burgess PW, Shallice T. The hayling and brixton tests. 1997.

75. Fonseca RP, Salles JFd, Parente MAdMP. Development and content validity of the Brazilian brief neuropsychological assessment battery Neupsilin. Psychology \& Neuroscience. 2008;1:55-62.

76. Hochstadt J. Set-shifting and the on-line processing of relative clauses in Parkinson's disease: Results from a novel eye-tracking method. Cortex. 2009;45:991-1011.

77. Cools R, Barker RA, Sahakian BJ, Robbins TW. Enhanced or impaired cognitive function in Parkinson's disease as a function of dopaminergic medication and task demands. Cerebral Cortex. 2001;11:1136-1143.

78. Hochstadt J, Nakano H, Lieberman P, Friedman J. The roles of sequencing and verbal working memory in sentence comprehension deficits in Parkinson's disease. Brain and language. 2006;97: 243-257.

79. Grossman M, Lee C, Morris J, Stern MB, Hurtig HI. Assessing resource demands during sentence processing in Parkinson's disease. Brain and language. 2002;80:603-616.

80. Skeel RL, Crosson B, Nadeau SE, Algina J, Bauer RM, Fennell EB. Basal ganglia dysfunction, working memory, and sentence comprehension in patients with Parkinson's disease. Neuropsychologia. 2001;39:962-971.

81. Grossman M, Zurif E, Lee C, Prather P, Kalmanson J, Stern MB, et al. Information processing speed and sentence comprehension in Parkinson's disease. Neuropsychology. 2002;16:174.

82. Lee C, Grossman M, Morris J, Stern MB, Hurtig HI. Attentional resource and processing speed limitations during sentence processing in Parkinson's disease. Brain and Language. 2003;85:347-356.

83. Colman KS, Koerts J, Stowe LA, Leenders KL, Bastiaanse R. Sentence comprehension and its association with executive functions in patients with Parkinson's disease. Parkinson's disease. 2011; 2011.

84. Zimmermann P, Fimm B. A test battery for attentional performance. Applied neuropsychology of attention Theory, diagnosis and rehabilitation. 2002:110-151.

85. Flowers K, Robertson C. The effect of Parkinson's disease on the ability to maintain a mental set. Journal of Neurology, Neurosurgery \& Psychiatry. 1985;48:517-529.

86. Stroop JR. Studies of interference in serial verbal reactions. Journal of experimental psychology. 1935;18:643.

87. Stinissen J, Willems P, Coetsier P, Hulsman W. Manual for the Dutch translated and adapted version of the Wechsler Adult Intelligence Scale (WAIS). Lisse: Swets and Zeitlinger; 1970.

88. Lelekov T, Franck N, Dominey PF, Georgieff N. Cognitive sequence processing and syntactic comprehension in schizophrenia. NeuroReport. 2000;11:2145-2149.

89. Breitenstein C, Van Lancker D, Daum I, Waters CH. Impaired perception of vocal emotions in Parkinson's disease: influence of speech time processing and executive functioning. Brain and cognition. 2001;45:277-314.

90. Daneman M, Carpenter PA. Individual differences in working memory and reading. Journal of verbal learning and verbal behavior. 1980;19:450-466.

91. Baddeley A. Exploring the central executive. The Quarterly Journal of Experimental Psychology: Section A. 1996;49:5-28.

92. Baddeley A. The central executive: A concept and some misconceptions. Journal of the International Neuropsychological Society. 1998;4:523-526. 